\title{
Optimismo disposicional y estilos de vida promotores de salud en estudiantes de Ciencias de la Salud
}

Dispositional Optimism and Health Promoters Lifestyles in Students of Health Sciences

\section{Loida Moya Smith}

Departamento de Enfermería de la Universidad de Alcalá (UAH). Alcalá de Henares, España.

Contacto: loidamos@gmail.com

Fecha de recepción: 11 de abril de 2017 / Fecha de aceptación: 27 de junio de 2017

\section{Resumen}

Objetivo principal: Determinar la correlación entre optimismo disposicional y los estilos promotores de salud de estudiantes universitarios de Ciencias de la Salud de la Universidad de Alcalá de Henares.

Metodología: Estudio descriptivo transversal. Se aplican los cuestionarios: Test de orientación vital revidado (LOT-R), para medir el optimismo disposicional y el cuestionario para medir estilos de vida promotores de salud y sus 6 dimensiones (HPLPII). La población final de estudio fue de 905 individuos, de los tres primeros cursos de Enfermería (55\%), Fisioterapia (15.8\%) y Medicina (29.2\%).

Resultados principales: En todas las correlaciones entre optimismo y estilos de vida promotores de salud se observó una correlación positiva significativa $(\mathrm{p}<0.01)$. Siendo más fuerte en crecimiento espiritual (el $23 \%$ de la variación se explica por el nivel de optimismo). En la escala de optimismo la puntuación media (M) es 14.78 y en la escala HPLPII es de 2.67, equivalente a un nivel moderado en ambos casos. En las subescalas del HPLPII los valores medios más bajos se dan en afrontamiento del estrés y responsabilidad en salud, con solo un $10 \%$ de la población que presenta con frecuencia o siempre estilos de vida promotores de salud. Se observan diferencias significativas por género. Los hombres tienen un nivel de optimismo mayor $(M=15.88)$ que las mujeres $(M=14.38)$, un nivel de EVPS global mayor (Hombres: $M=142.14$; Mujeres: $M=137.64$ ).

Conclusión principal: Ser optimista está correlacionado con llevar un estilo de vida más saludable. Los resultados también sugieren que los estudiantes universitarios de ciencias de la salud no realizan los hábitos saludables de forma rutinaria o siempre, considerándose una conducta de riesgo para la salud. Por último, destacar que los hombres obtienen mejores resultados tanto en el nivel de optimismo, como en la variable estilo de vida promotor de salud global y sus subescalas, exceptuando las relaciones interpersonales. Estos resultados pueden tener implicaciones importantes en futuras intervenciones relativas a la salud.

Palabras clave: optimismo disposicional, estudiantes universitarios, estilo de vida promotor de salud, responsabilidad en salud, actividad física, nutrición, manejo del estrés, relaciones interpersonales y crecimiento espiritual.

\footnotetext{
Abstract

Objective: Determine the correlation between dispositional optimism and healthy lifestyles in students in the Health Sciences Program at Alcala de Henares University.

Methods: Cross-sectional study. The Life Orientation Test-Revised (LOT-R) was used to measure dispositional optimism and the Health-Promoting Lifestyle Profile (HPLPII) questionnaire, was used to measure the mean score of the global dimension as well as the six individual dimensions of healthy lifestyles. The study population consisted of 905 individuals from the first three years of the following programs: nursing (55\%), physical therapy (15.8\%) and medicine (29.2\%).

Results: A significant positive correlation $(\mathrm{p}<0.01)$ was observed between optimism and healthy lifestyles dimensions. The correlation was weaker between optimism and three dimensions: the global dimension, interpersonal relationships and stress management. The correlation was stronger between optimism and spiritual growth ( $23 \%$ of the variation is explained by the level of optimism). The optimism mean score (M) is 14.78 and in HPLPII scale is 2.67 , equivalent in both cases to a moderate level. In the subscales of HPLPII the lowest values occur in stress management and health responsibility. Only $10 \%$ of the population has often or always HPLP. Significant differences are
} 
observed by gender. Men have a higher level of optimism $(M=15.88)$ than women $(M=14.38)$, a higher level of the global dimension of health-promoting lifestyle (men: $M=142.14$; women: $M=137.64$ ).

Conclusions: Being optimistic is correlated with leading a healthier lifestyle. The results also suggest that Health Science students, only sometimes perform healthy habits. This is considered insufficient. Finally, note that men get better results at both, level of optimism and the global health-promoting lifestyle dimension and the same for the subscales, except for interpersonal relationships. These results may have important implications for future interventions on health.

Keywords: dispositional optimism, college students, health promoting lifestyle, health responsibility, physical activity, nutrition, stress management, interpersonal relationships and spiritual growth.

\section{Introducción}

La promoción de salud es un factor determinante del estado de salud individual, teniendo en cuenta que la persona es responsable de su propia salud ${ }^{1}$. Es un concepto holístico, entendido desde un punto de vista global del ser humano, que ha ido cambiando, desde una visión más paternalista, hacia otra perspectiva más dirigida hacia el empoderamiento del individuo y la comunidad favoreciendo las opciones personales y grupales para mejorar el bienestar' ${ }^{2}$.

El hecho de que se considere a la salud desde una perspectiva más amplia ha aumentado las políticas de promoción de hábitos de vida saludable, concepto que se ha ido transformando. Concretamente, la Organización Mundial de la Salud, a través de la Carta de Ottawa para la Promoción de la Salud, sentó las bases para fomentar estrategias de salud. Inicialmente se incluyó en este concepto a escuelas, hogares, lugares de trabajo y el ámbito comunitario como impulsores de estilos de vida saludable, pero más recientemente se han añadido a esta lista otros entornos como las universidades ${ }^{3}$. Dentro del entorno universitario también se impulsan estrategias de promoción de la salud dirigidas hacia los futuros profesionales sanitarios y su entorno de trabajo, así como para el cuidado del paciente ${ }^{4}$. Para aumentar el compromiso con aquellas prácticas promotoras de salud a nivel social, profesional y personal, es necesario crear ambientes promotores de la salud en todos los ámbitos.

Una de las etapas de la vida que se considera más importante para la adquisición de buenos hábitos de salud es la etapa universitaria ${ }^{5}$. Diversos autores sostienen que el acceso a la universidad supone un cambio importante en el individuo, que puede determinar que los estilos de vida se vean modificados por cambios de tipo social, económico, cultural y psicológico a los que deberán enfrentarse durante su proceso de formación.
Todo esto puede originar diferentes problemas que se reflejen en el estado de salud y que a su vez puedan repercutir en su rendimiento académico ${ }^{6}$.

Un factor importante que tiene una gran influencia sobre el afrontamiento de los problemas es el optimismo, existiendo un número considerable de estudios que han investigado sobre esta cuestión ${ }^{7-10}$, llegando a la conclusión de que, el optimismo disposicional, supone un recurso muy valioso a nivel psicológico que puede llegar a ser especialmente importante, cuando las personas se enfrentan a situaciones de riesgo o eventos que amenazan su bienestar.

De hecho, en investigaciones recientes, ha habido sustanciales progresos en cuanto a la contribución del optimismo sobre un buen estado de salud y alta calidad de vida ${ }^{6,8,11,12}$. Sin embargo, no existen muchos estudios sobre la influencia del optimismo en las diferentes variables que influyen en los estilos de vida de estudiantes universitarios. Resaltar, además, que existe un vínculo importante entre el trabajo realizado por el personal sanitario, que ha incorporado en su vida estilos de vida saludables, y la mejora de la salud del paciente ${ }^{13}$. Sin embargo, muchos futuros profesionales sanitarios a menudo están trabajando en ambientes que no promueven la salud ${ }^{14}$.

En este sentido, Nola Pender ${ }^{1}$ se interesó por un modelo enfermero que diera respuestas a la forma en la que las personas adoptan decisiones acerca del cuidado de su propia salud. En este modelo se estudian seis dimensiones: actividad física, nutrición, manejo del estrés, responsabilidad en salud, relaciones interpersonales y crecimiento espiritual o autorrealización ${ }^{15}$. A todo esto hay que añadir que investigaciones recientes demuestran la contribución del optimismo sobre un buen estado de salud y alta calidad de vida ${ }^{6,8,11,12,16}$. De ahí la importancia de analizar la influencia del optimismo sobre estilos de vida promotores de salud. 
Muchas otras investigaciones también corroboran la correlación entre optimismo disposicional y el hecho de disfrutar de un mejor estado de salud además de mayor calidad de vida; por ejemplo, Carver et $_{\text {al }}{ }^{17}$, estudian cómo influye el optimismo, en el afrontamiento de la angustia, en un grupo de mujeres con cáncer de mama en su estadio inicial. Llegando a la conclusión de que el optimismo se relacionaba inversamente con la angustia, incluso controlando la angustia previa al diagnóstico. Otros autores como Kim et al. ${ }^{16}$ concluyeron que el optimismo disposicional previene, en ancianos, los accidentes cerebrovasculares. Este estudio difiere de otros en los que se analizó la influencia del bienestar del individuo sobre la salud. En dichas investigaciones, se entiende el bienestar de una forma muy amplia, incluyendo variables como vitalidad, satisfacción personal o afectos positivos además del optimismo ${ }^{18}$. De tal forma que, al no analizar dimensiones específicas, es difícil saber si es solo el optimismo el que produce dichos resultados de salud o son otras variables.

El optimismo disposicional ha sido definido como una esperanza generalizada de que cosas buenas suce$d^{10}{ }^{10}$. Estas expectativas se mantienen relativamente estables a través del tiempo y el contexto, teniendo influencia no solo en las emociones individuales sino en las decisiones, mediante la lucha o la renuncia ${ }^{19}$. Los optimistas ven que los resultados deseados están a su alcance, por lo que es más probable que continúen luchando, para conseguir dichos resultados, incluso cuando sea difícil o doloroso, al contrario que los pesimistas $^{20}$.

La pertinencia de este estudio radica en que no existe constancia de ninguna investigación que haya vinculado el optimismo disposicional y las 6 dimensiones del modelo de promoción de la salud de Pender' ${ }^{2}$ nutrición, crecimiento espiritual, actividad física, relaciones interpersonales, manejo del estrés, responsabilidad en salud y estilo de vida promotor de salud global. Aunque existe evidencia de trabajos que vinculan al optimismo disposicional con algunas de estas 6 variables ${ }^{7,8,16,21}$, pero en ningún caso, según la bibliografía revisada, se ha vinculado con el modelo de promoción de salud.

\section{Objetivo}

El objetivo del presente estudio va a consistir en analizar la relación entre OD y el modelo de promoción de la salud en estudiantes universitarios de Medicina,
Enfermería y Fisioterapia, teniendo en cuenta las posibles variaciones producidas por las variables demográficas.

\section{Metodología}

En este estudio se pretende conocer la correlación entre optimismo disposicional y las dimensiones de los estilos de vida promotores de salud, además de la frecuencia de comportamientos saludables, según el modelo de promoción de salud, en estudiantes universitarios de Enfermería, Fisioterapia y Medicina de 1. ${ }^{\circ}$, $2 .^{\circ}$ y $3 .^{\text {er }}$ curso. Para la consecución de este fin, se ha diseñado un estudio observacional, transversal y descriptivo. El estudio se ha realizado desde enero a junio de 2015.

\section{Muestra}

La población final de estudio consta de 905 individuos que completaron el cuestionario en la Universidad de Alcalá, de algunos de los tres primeros cursos de las carreras de Enfermería (55\% de la población), Fisioterapia (15.8\%) o Medicina (29.2\%). Para los fines del estudio, se ha diferenciado a los estudiantes de enfermería por campus, representando los del campus de Alcalá el $32 \%$ de la población frente al $23 \%$ de los que estudian en el campus de Guadalajara. Los estudiantes son mayoritariamente de género femenino $(73.1 \%$ del total) y solteros (93\%) y tienen una edad menor a 26 años $(94.3 \%)$, siendo el rango de edad más frecuente el de 20-25 años (53.8\%). El reparto entre cursos es relativamente homogéneo, suponiendo los estudiantes de primero, segundo y tercero un $36.2 \%, 31.8 \%$ y $31.9 \%$ respectivamente.

\section{Instrumentos de medición}

Dos fueron las escalas de medida empleadas: para medir el nivel de optimismo disposicional se utilizó el Test de Orientación Vital revisado LOT- $\mathrm{R}^{22}$ en la versión española ${ }^{23}$; para medir los estilos de vida promotores de salud se empleó el cuestionario Health-Promoting Lifestyle Profile [HPLP II] (Adult Version) ${ }^{24}$ publicado en español ${ }^{25}$. En cuanto al Test de Orientación Vital, se ha utilizado el revisado ${ }^{22} \sin l o s 4$ ítems de relleno, para no hacer tan extenso el cuestionario final y facilitar la cumplimentación de los cuestionarios a los participantes. La forma de puntuación se mantuvo igual que en la primera versión. Referente al Lifestyle Profile II (HPLPII) ${ }^{24}$, está compuesto por 52 ítems que corresponden a una escala total, además de seis subes- 
calas para medir los comportamientos que promocionan los estilos de vida saludables.

\section{Análisis estadístico}

El procesamiento y análisis de los datos se realizó con los programas estadísticos R v.3.2.0 y SPSS v.20.

En cuanto a las variables resultado extraídas del cuestionario, vienen dadas como respuestas tipo Likert con cuatro (cuestionario LOTR) o cinco (cuestionario HPLPII) valores posibles. Siguiendo las recomendaciones de la bibliografía ${ }^{26,27}$ se han definido como variables cuantitativas de intervalo para los análisis inferenciales.

Además, se trabajó con los valores globales de la escala de Estilos de Vida saludable (EVS) y sus subdimensiones en el análisis inferencial. Los valores medios fueron utilizados en el análisis descriptivo y en las representaciones gráficas del análisis inferencial, para facilitar su interpretación y comparación.

En el análisis descriptivo se extrajeron medidas de frecuencia en las variables categóricas (sociodemográficas) y medidas de tendencia central, dispersión y simetría (media, intervalo de confianza, media recortada al $5 \%$, mediana, varianza, desviación típica, valores mínimos y máximos, asimetría y curtosis) en las variables de intervalo (resultados de los test).

Para evaluar la normalidad de la distribución de las variables, se emplearon métodos visuales (histograma, gráficos boxplot y Q-Q') y se aplicaron los test de normalidad de Kolmogórov-Smirnov, Shapiro-Wilk, Shapiro-Francia, Cramer-von Mises y AndersonDarling. Dado el elevado tamaño muestral (n>900) y el cumplimiento por parte de las variables de las condiciones del teorema del límite central ${ }^{28,29}$, se decidió la realización de pruebas paramétricas. Además, hay que destacar la robustez de las pruebas empleadas (ANOVA y t de Student) ante la violación del supuesto de normalidad.

El estudio de las correlaciones bivariadas entre el optimismo y el EVS y sus dimensiones, se realizó mediante análisis de regresión lineal simple, comprobándose el cumplimiento de la linealidad mediante el estadístico F de Snedecor. Se determinaron los coeficientes de correlación de Pearson y de determinación.
Para el estudio de la influencia del género se utilizó el estadístico t de Student para muestras independientes y el estudio de la influencia de la edad, titulación y curso se realizó mediante ANOVA de un factor, con análisis previo de homocedasticidad mediante la prueba de Levene para todos los casos. Se realizó análisis post-hoc mediante las pruebas de Turkey y en tamaños de grupos desiguales, se utilizó la prueba de Gabriel. En los casos donde no se cumplió el principio de homocedasticidad, se utilizó la prueba de Welch y para el análisis post-hoc el estadístico Games-Howell.

El análisis de correlación entre las seis dimensiones que definen el EVS se llevó a cabo mediante regresión lineal con el coeficiente de correlación de Pearson.

En todos los casos se asumió un nivel de significación estadística del $95 \%(\mathrm{p}<0.05)$, excepto en el análisis de correlación de las seis dimensiones de EVS en el que fue del $99 \%(\mathrm{p}<0.01)$.

\section{Resultados}

En cuanto a los resultados, la puntuación media (M) en la escala de optimismo es 14.78 , correspondiente a un nivel de optimismo moderado, con una desviación estándar (DE) de 4.54 [gráfica 1]. El estudio de los cuantiles muestra que sólo un $10 \%$ (percentil 10=10) de la población muestra valores de optimismo bajos o muy bajos, frente a un $40 \%$ (percentil $60=17$ ) que muestra valores altos o muy altos.

En la escala de EVS y sus subescalas se recomienda utilizar los valores medios para que puedan ser comparables entre sí. Así, el resultado medio de la escala HPLPII en la población [gráfica 4] es de 2.67 (DE 0.34 ), equivalente a una participación moderadamente frecuente en actividades saludables. Sólo un $2.5 \%$ de la población está por debajo del valor de corte 2 ("a veces"), equivalente a realizar con poca frecuencia o nunca actividades relacionadas con EVS. En el otro extremo encontramos el punto de corte 3 ("con frecuencia") en el percentil 82.5, es decir, sólo un $17.5 \%$ de la población realiza con frecuencia o siempre actividades relacionadas con EVS.

En las subescalas de HPLPII [gráfica 2-9] encontramos los valores medios más elevados en "relaciones interpersonales" $(\mathrm{M}=3.18 ; \mathrm{DE}=0.48)$ y "crecimiento espiritual" ( $\mathrm{M}=3.05 ; \mathrm{DE}=0.49)$. En ambas escalas hay un $97.5 \%$ de la población con valores por encima de 2 , dándose un $62.5 \%$ (crecimiento espiritual) y $70 \%$ 
(relaciones interpersonales) de la población con valores por encima de 3 .

En el otro extremo, los valores medios más bajos se dan en "afrontamiento del estrés" $(\mathrm{M}=2.34 ; \mathrm{DE}=0.47)$ y "responsabilidad en salud" (M=2.36; DE 0.51). En la primera subescala, un $20 \%$ de la población muestra valores por debajo de 2 ("a veces"), con solo un $10 \%$ (percentil 90) de la población que presenta con frecuencia o siempre EVS (valores por encima de 3). En el caso de la responsabilidad en salud, encontramos un $20 \%$ por debajo del valor 2 , y un $13.5 \%$ de la población con valores por encima de 3 .

La subescala de "actividad física" tiene una media de 2.4 (DE 0.71), con un 30\% de la población por debajo del valor 2 y un $25 \%$ de la población (percentil 75) por encima del valor de corte 3 . En el caso de la subescala de "nutrición" $(\mathrm{M}=2.62 ; \mathrm{DE}=0.48)$, sólo un $10 \%$ de la población está por debajo del valor de corte 2 , frente a un $25 \%$ (percentil 75 ) por encima del valor 3 , es decir, que presentan con frecuencia o siempre EVS en relación a la nutrición.

Nivel de optimismo y EVS. Se realizó el estudio de las correlaciones entre el nivel de optimismo y EVS, tanto del EVS global como de las seis dimensiones que lo componen. Se optó por la técnica de regresión lineal simple. En general, se observa un mal ajuste de la recta a la nube de puntos. Los niveles críticos del estadístico $\mathrm{F}$ de Snedecor (valores en todos los casos de $\mathrm{p}<0.01$ ) indicaron que los pares de variables estaban linealmente relacionados. En todas las correlaciones realizadas se observó una correlación positiva significativa $(\mathrm{p}<0.01)$, con una recta ascendente en los diagramas de dispersión, de poca pendiente. El nivel de correlación del optimismo es bajo en tres dimensiones: EVS global $(\mathrm{R}=0.370)$, relaciones interpersonales $(\mathrm{R}=0.310) \mathrm{y}$ manejo del estrés $(\mathrm{R}=0.279)$. La correlación del optimismo y responsabilidad en salud es muy baja $(\mathrm{R}=0.108)$, al igual que ocurre con la actividad física $(\mathrm{R}=0147)$ y la nutrición $(\mathrm{R}=0.194)$. La correlación más fuerte, con un nivel moderado, se da entre el optimismo y el crecimiento espiritual $(\mathrm{R}=0.480)$.

El 13.7\% $(\mathrm{R} 2=0.137)$ de la variación de EVS global está explicada por el optimismo. A su vez, el optimismo influencia en un $1.2 \%$ a la responsabilidad en salud ( $R 2=0.012$ ), en un $2.2 \%$ a la actividad física $(\mathrm{R} 2=0.022)$, en un $3.8 \%$ al nivel nutricional $(\mathrm{R} 2=0.038)$, en un $9.6 \%$ a las relaciones interpersonales $(\mathrm{R} 2=0.096)$ y en un $7.8 \%$ al manejo del estrés
$(\mathrm{R} 2=0.078)$. Destaca el hecho de que el $23 \%$ $(\mathrm{R} 2=0.230)$ de la variación en el crecimiento espiritual se explica por el nivel de optimismo.

Hay que tener en cuenta que el hecho de que las correlaciones sean significativas puede estar condicionada por un tamaño muestral alto [gráfica 3].

Influencia del género en el optimismo y EVS. Se estudió el optimismo, EVS global y cuatro de sus dimensiones (nutrición, actividad física, relaciones interpersonales y manejo del estrés) en función del género del estudiante. Tanto este análisis como los siguientes (edad, titulación y curso) muestran un número de sujetos diferentes por nivel, tratándose pues de diseños no equilibrados.

Para todos los casos, se estudió el principio de homocedasticidad mediante prueba de Levene, dando un valor no significativo para el estadístico F de Snedecor, lo que demuestra igualdad de varianza en los grupos.

Se observan diferencias significativas por género, en el nivel de optimismo $(\mathrm{t}=4.418 ; \mathrm{p}<0.05)$, EVS global $(\mathrm{t}=3.327 ; \mathrm{p}<0.05)$, nutrición $(\mathrm{t}=2.168 ; \mathrm{p}<0.05)$, actividad física $(\mathrm{t}=4.983 ; \mathrm{p}<0.05)$, relaciones interpersonales $(\mathrm{t}=-2.020 ; \mathrm{p}<0.05)$ y manejo del estrés $(\mathrm{t}=3.738 ; \mathrm{p}<0.05)$. Así, los hombres tienen un nivel de optimismo mayor $(\mathrm{M}=15.88$; IC95 \% $=15.32$, 16.45; $\mathrm{DE}=0.288)$ que las mujeres $(\mathrm{M}=14.38$; IC95 \% $=14.04$, 14.73; DE=0.176), un nivel de EVS global mayor (Hombres: $\mathrm{M}=142.14$; IC95 \%=139.87, 144.41; $\mathrm{DE}=1.15$; Mujeres: $\mathrm{M}=137.64$; $\mathrm{IC} 95 \%=136.27$, 139.01; $\mathrm{DE}=0.698)$, una nutrición más saludable (Hombres: $M=24.11$; IC95 \%=23.53, 24.70; $\mathrm{DE}=0.296$; Mujeres: $\mathrm{M}=23.40$; IC95 \% $=23.07,23.73$; $\mathrm{DE}=0.166$ ), mayor nivel de actividad física (Hombres: $\mathrm{M}=21.07$; $\mathrm{IC} 95 \%=20.32,21.82 ; \mathrm{DE}=0.380$; Mujeres: $\mathrm{M}=18.55$; $\mathrm{IC} 95 \%=18.14,18.97 ; \mathrm{DE}=0.212$ ) y mayor manejo del estrés (Hombres: $M=19.47$; IC95 \%=19.01, 19.94; $\mathrm{DE}=0.235$; Mujeres: $\mathrm{M}=18.43$; IC95 $\%=18.14,18.71 ; \mathrm{DE}=0.145$ ). Por el contrario, son las mujeres las que presentan mayor nivel de relaciones interpersonales ( $M=28.83$; IC95\%=28.50, 29.6; $\mathrm{DE}=0.168)$ respecto a los hombres $(\mathrm{M}=28.18$; IC95 \% $=27.65,28.71 ; \mathrm{DE}=0.269$ ) [gráfica 4].

Influencia de la titulación en el optimismo y EVS. El análisis ANOVA indicó diferencias significativas por titulación en el nivel de optimismo $(\mathrm{F}=6.878, \mathrm{p}<0.05)$ $\mathrm{y}$ en el nivel de actividad física $(\mathrm{F}=3.468 ; \mathrm{p}<0.05)$ [gráfica 5]. 
Las comparaciones múltiples realizadas mediante prueba de Turkey indican, con significación estadística, que los estudiantes de Medicina $(\mathrm{M}=15.57$; IC95\% $=15.00,16.15 ; \mathrm{DE}=0.292$ ) y los de Fisioterapia $(\mathrm{M}=15.06$; IC95 \%=14.36, 15.75; $\mathrm{DE}=0.350)$ tienen un nivel de optimismo mayor que los de Enfermería Alcalá $(\mathrm{M}=13.87$; IC95\%=13.34, 14.40; $\mathrm{DE}=0.270)$. Por otro lado, tanto la prueba de Gabriel como la de Turkey indican que los estudiantes de Fisioterapia $(\mathrm{M}=20.17$; IC95 \%=19.22, 21.13; $\mathrm{DE}=0.482)$ tienen un nivel de actividad física significativamente mayor que los de Enfermería Alcalá ( $M=18.42$; IC95 \% $=17.75,19.09 ; \mathrm{DE}=0.340)$.

En los dos casos de no igualdad en las varianzas, la prueba de Welch no indicó diferencias significativas [gráfica 6].

Influencia de la edad en el optimismo y EVS. El análisis ANOVA indicó diferencias significativas por edad en el nivel de optimismo $(\mathrm{F}=3.047, \mathrm{p}<0.05)$, en el nivel de actividad física $(\mathrm{F}=4.645 ; \mathrm{p}<0.05)$ y en el de relaciones interpersonales $(\mathrm{F}=3.259, \mathrm{p}<0.05)$. El análisis Welch no indicó diferencias significativas [gráfica 7].

Las comparaciones múltiples realizadas mediante prueba de Gabriel indican, con significación estadística, que los estudiantes de 31 años y más $(\mathrm{M}=16.59$; IC95\% $=15.02,18.16 ; \mathrm{DE}=0.771$ ) tienen un nivel de optimismo mayor que los de 19 años y menores $(\mathrm{M}=14.37 ; 13.90,14.84 ; \mathrm{DE}=0.238)$. Sin embargo, dichos estudiantes de 31 años o más $(M=16.15$; IC95 \% $=14.21,18.08 ; \mathrm{DE}=0.952$ ) tienen un nivel de actividad física menor que los de 19 años o menos $(\mathrm{M}=19.67 ; 19.09,20.24$; $\mathrm{DE}=0.291)$ y que los de 2025 años $(\mathrm{M}=19.14$; IC95 \%=18.63, 19.65; $\mathrm{DE}=0.260)$.

Por último, son los estudiantes de entre 26-30 años $(\mathrm{M}=26.06$; IC95 \%=23.82, 28.29; $\mathrm{DE}=1.059)$ los que tienen un nivel de relaciones interpersonales significativamente menor que los de 19 o menos años $(\mathrm{M}=28.44 ; \mathrm{IC} 95 \%=27.99,28.89 ; \mathrm{DE}=0.231)$ y que los de 20-25 años $(\mathrm{M}=28.94$ : $\mathrm{IC} 95 \%=28.57,29.31$; $\mathrm{DE}=0.189$ ) [gráfica 8].

Influencia del curso en el optimismo y EVS. El análisis ANOVA no indicó diferencias significativas por curso. La prueba de Welch indicó diferencias significativas para la dimensión EVS global $(\mathrm{W}=3.440$, $\mathrm{p}<0.05)$ y para el manejo del estrés $(\mathrm{W}=5.573, \mathrm{p}<0.05)$ [gráfica 9].
Las comparaciones múltiples realizadas mediante prueba de Games-Howell indican, con significación estadística, que los estudiantes de $1 .^{\text {er }}$ curso $(\mathrm{M}=136.94 ; \quad \mathrm{IC} 95 \%=135.08,138.80 ; \mathrm{DE}=0.944)$ tienen un nivel de EVS global menor que los de 2. ${ }^{\circ}$ curso $(M=140.52 ; \quad$ IC95 $\%=138.50,142.55$; $\mathrm{DE}=1.027)$ y que el nivel de manejo del estrés de los estudiantes de 1 er $^{\text {er }}$ curso $(\mathrm{M}=18.21$; IC95 $\%=17.84$, 18.59; $\mathrm{DE}=0.191)$ es menor que los de $2 .^{\circ}$ curso $(\mathrm{M}=19.14 ; \mathrm{IC} 95 \%=18.72,19.55 ; \mathrm{DE}=0.209)$ [gráfica 10].

Correlaciones entre las dimensiones que determinan el EVS. Se procedió al estudio de las seis dimensiones que definen el EVS y sus relaciones entre sí. Todas las dimensiones que componen el EVS se correlacionan entre sí de forma significativa, según el coeficiente $\mathrm{R}$ de Pearson, a un nivel de significación del $99 \%$. La mayoría de las correlaciones son positivas y bajas ( $R$ situado entre los valores 0.223 y 0.391 ), habiendo una correlación positiva muy baja $(\mathrm{R}=0.147)$ entre actividad física y relaciones interpersonales. Destacan tres correlaciones positivas de intensidad moderada entre crecimiento espiritual y manejo del estrés $(R=0.482)$, entre crecimiento espiritual y relaciones interpersonales $(\mathrm{R}=0.557)$ y entre actividad física y nutrición $(\mathrm{R}=0.402)$ [gráfica 11].

\section{Discusión}

A lo largo de las últimas dos décadas, se han realizado numerosas investigaciones para estudiar el nivel de optimismo y los estilos de vida promotores de salud en estudiantes universitarios, pero no se han encontrado estudios que busquen una correlación entre ambos utilizando el modelo de promoción de salud. En esta investigación se ha hecho un estudio preliminar centrado en avanzar en el estudio de dicha correlación, teniendo en cuenta que el optimismo disposicional es una variable fundamental en la salud mental y física del individuo $^{10}$. Los hallazgos encontrados, respecto a la puntuación media en la escala de optimismo, correspondieron a un nivel de optimismo moderado, que coincidió con los resultados de la escala de HPLPII. En este estudio en todas las correlaciones realizadas entre optimismo y EVPS, se observó una correlación positiva significativa $(\mathrm{p}<0,01)$. Al igual que en el estudio de Rasmussen $^{26}$, en el que se realizó un metaanálisis con una revisión sistemática de 83 estudios, en los que se analizaba la correlación entre optimismo y la salud física, obteniendo una correlación positiva significativa $(p<0,01)$ y concluyendo que el optimismo es un 
predictor significativo del estado de nuestra salud física $^{21}$. En los resultados también se encontró que el optimismo tiene una influencia del $13,7 \%$ sobre la variación de estilos de vida promotores de salud, lo que coincide con otros estudios realizados en este senti$\mathrm{do}^{8,30}$. En los que se llega al mismo argumento, relativo a que los individuos optimistas, abordan la promoción de la salud de una forma más activa. Siendo más probable que realicen ejercicio físico, lleven dietas adecuadas y eviten conductas poco saludables. Muchas otras investigaciones, también corroboran la correlación entre optimismo disposicional y el hecho de disfrutar de una mejor salud, además de mayor calidad de $\operatorname{vida}^{8,30}$.

También se llegó a la conclusión de que los estudiantes con mayores niveles de optimismo disposicional realizan más actividad física. Encontramos que existe una correlación positiva, pero de baja magnitud, entre optimismo y actividad física. Estos resultados apoyan previas investigaciones en las que se ha observado que los optimistas muestran un mayor compromiso en programas de entrenamiento físico ${ }^{16}$. Además, se obtiene una correlación muy baja, pero estadísticamente significativa, entre optimismo disposicional y nivel nutricional. Resultados similares se obtuvieron en otro estudio que se realizó en adultos jóvenes finlan$\operatorname{deses}^{31}$, en el que se concluyó que el optimismo mejora los hábitos dietéticos. Concretamente se observó que los individuos más optimistas llevaban dietas más saludables y controlaban el peso de forma más eficaz ${ }^{31}$.

Durante las dos últimas décadas algunos investigadores han incluido la espiritualidad como una dimensión del bienestar y a pesar de la diversidad de significados, entre los diferentes autores, existen algunos puntos en común. Éstos, incluyen el sentido positivo de una vida con significado y propósitos, conectados a uno mismo, al medio ambiente o a un poder superior. En un estudio, realizado a estudiantes universitarios de Ciencias de la Salud de la Universidad de Texas, se encontró que aquellos con una perspectiva más positiva tenían más propósitos y objetivos en sus vidas, lo que mejoraba la sensación de bienestar general ${ }^{32}$. Resultados que coinciden con los de nuestra investigación, ya que existe una correlación positiva significativa y con un nivel moderado entre optimismo y crecimiento espiritual. Destaca el hecho de que el $23 \%$ de la variación en el crecimiento espiritual, se explica por el nivel de optimismo. Estos resultados resaltan la importancia de las percepciones subjetivas como indicadores válidos de futuros objetivos de salud ${ }^{16,33}$. También se ha investigado a cerca del optimismo como un regulador del estrés. En un estudio realizado a estudiantes universitarios afroamericanos, en el que se investigó optimismo y estrés, se demuestra la evidencia empírica de que la percepción de un individuo del estrés puede estar influida por el constructo de la personalidad, optimismo. Además, el optimismo obtuvo una correlación significativa, negativamente relacionada con los niveles de estrés percibido ${ }^{34}$. Nuestros resultados son coherentes con este y otros estudios que investigan el optimismo disposicional como moderador del estrés $^{35,36}$.

En cuanto al resultado medio de la escala de HPLPII en la población estudiada, correspondió a una participación moderadamente frecuente en actividades saludables, es decir, cercanos a la frecuencia considerada como saludable, según el modelo de Nola Pender. Estos resultados son similares a los obtenidos en otros estudios como el que se realizó en China o Colom$\mathrm{bia}^{37,38}$, donde la puntuación media de las dimensiones del HPLPII se encontraban en un nivel medio. Estos resultados difieren de los obtenidos por Triviño et al. ${ }^{39}$, en el que se obtuvieron resultados superiores a la media

Por último, comentar la influencia de los factores sociodemográficos en el optimismo y los estilos de vida promotores de salud. En nuestro estudio se han observado diferencias significativas por género, en el nivel de optimismo. Así los hombres tienen un nivel de optimismo mayor que las mujeres. Además, el sexo masculino también tiene un Estilo de vida saludable global mayor. Esto concuerda con otros estudios como el de Vidal et al $^{40}$. Sin embargo, no es congruente con lo encontrado por otros autores como Cid et al. ${ }^{41}$, en el que los hombres tienen promedios más bajos respecto a las mujeres.

En relación con la edad, en nuestro estudio, los estudiantes entre 26 y 30 años tienen un nivel de relaciones interpersonales significativamente menor que los de 20 años y menores, estos resultados son consistentes con los obtenidos por Abeer et al. ${ }^{42}$ en el que se correlaciona negativamente la edad de los estudiantes con las relaciones interpersonales.

\section{Conclusiones}

El interés de esta investigación se encuentra en el análisis del nivel de OD de los estudiantes universitarios, y su relación con las 6 dimensiones del modelo de promoción de la salud. El hallazgo más importante de este 
trabajo viene dado por los resultados que se derivan de la combinación de dos instrumentos utilizados, el LOT-R y el HPLP II, que no se habían empleado anteriormente en un mismo estudio, como se ha podido constatar tras una exhaustiva revisión bibliográfica.

En base a la hipótesis planteada, hemos obtenido el hallazgo más importante de este estudio, en el que se demuestra que el OD está positivamente relacionado con estilos de vida promotores de salud, es decir, los alumnos más optimistas participan con más frecuencia en conductas promotoras de salud.

Los resultados obtenidos también indican que la mayoría de los estudiantes de este estudio tienen un adecuado estilo de vida en términos de promoción de salud. El 81 \% de la población a estudio tiene una participación moderadamente frecuente en actividades saludables, lo que se considera aceptable. Pero, a pesar de estos resultados, se deberían realizar más intervenciones para la mejora de los estilos promotores de salud en estudiantes universitarios, ya que tan solo un $17.5 \%$ de la población realiza con frecuencia o siempre actividades relacionadas con estilos de vida promotores de salud. Además, los valores medios más bajos se encontraron en las variables afrontamiento del estrés y responsabilidad en salud, dos variables fundamentales para el mantenimiento de la salud a largo plazo y para la adquisición de buenos hábitos.

Destacar también que se encontraron diferencias significativas por género en el nivel de optimismo y estilo de vida promotor de salud. Así, los hombres tienen un nivel de optimismo disposicional, estilo de vida saludable global, nutrición, actividad física y manejo del estrés mayor que las mujeres. Por el contrario, son las mujeres las que presentan mayor nivel de relaciones interpersonales. De este resultado se puede deducir la necesidad de intervención sobre la población femenina de estudiantes universitarias de Ciencias de la Salud para mejorar el nivel de optimismo y el estilo de vida.

También se puede destacar que los estudiantes de Fisioterapia tienen un nivel de optimismo disposicional y de actividad física mayor que los de Enfermería de Alcalá. Este resultado continua con estudios anteriores, en lo que se relacionan mayores niveles de optimismo con mayores niveles de actividad física. De esto se puede deducir la importancia de avanzar en este estudio, a través de un estudio longitudinal, donde se pueda analizar la importancia del optimismo sobre la variable actividad física en los estudiantes de Enfermería, Fisioterapia y Medicina.

Por último, destacar que los estudiantes de 1 er $^{\text {cr }}$ curso tienen un nivel de estilo de vida saludable global y manejo del estrés menor que los de $2 .^{\circ}$ curso, lo que también nos indica que el primer año de universidad requiere de un proceso de adaptación en el que puede ser necesario aprender a manejar y solucionar de forma adecuada situaciones estresantes.

\section{Bibliografía}

1. Pender NJ. Heath Promotion Model Manual. 2011.

2. Pender NJ, Murdaugh CL, Parsons MA. Health promotion in nursing practice. 2006

3. Dooris M, Doherty S, Cawood J, Powell S. The healthy universities approach: adding value to the higher education sector. Health Promotion Settings: Principles and Practice 2011:153.

4. Cancela Carral JM, Ayán Pérez C. Prevalencia y relación entre el nivel de actividad física y las actitudes alimenticias anómalas en estudiantes universitarias españolas de ciencias de la salud y la educación. Revista Española de Salud Pública 2011;85(5):499505.

5. Hernán M, Fernández A, Ramos M. La salud de los jóvenes. Gaceta Sanitaria 2004; 18:47-55.

6. Carver CS, Scheier MF, Segerstrom SC. Optimism. Clin Psychol Rev 201011;30(7):879-889.

7. Scheier MF, Carver CS. Optimism, coping, and health: assessment and implications of generalized outcome expectancies. Health psychology 1985;4(3):219.

8. Carver CS, Scheier MF. Dispositional optimism. Trends Cogn Sci (Regul Ed) 2014;18(6):293-299

9. Scheier MF, Carver CS. Effects of optimism on psychological and physical well-being: Theoretical overview and empirical update. Cognitive therapy and research 1992;16(2):201-228.

10. Scheier MF, Carver CS. Optimism, coping, and health: assessment and implications of generalized outcome expectancies. Health psychology 1985;4(3):219.

11. Peterson C. The future of optimism. Am Psychol 2000;55(1):44

12. Song ZY. Relations between optimism, stress and health in Chinese and American students. 2003

13. Baumann A, Giovannetti P, O'Brien-Pallas L, Mallette C, Deber R, Blythe J, et al. Healthcare restructuring: the impact of job change. Can J Nurs Leadersh 2001 Jan-Feb;14(1):14-20.

14. Pender N, Murdaugh G, Parsons M. Social support and health promotion. Health Promotion in Nursing Practice.5th ed. New Jersey: Pearson Education, Inc 2006:223-242.

15. Walker, S.N., \& Hill-Polerecky, D.M. Psychometric evaluation of the Health-Promoting Lifestyle Profile II. 1996.

16. Kim ES, Park N, Peterson C. Dispositional optimism protects older adults from stroke. AHA Stroke 2011.

17. Carver CS, Pozo C, Harris SD, Noriega V, Scheier MF, Robinson DS, et al. How coping mediates the effect of optimism on distress: a study of women with early stage breast cancer. J Pers Soc Psychol 1993;65(2):375.

18. Chida Y, Steptoe A. Positive psychological well-being and mortality: a quantitative review of prospective observational studies. Psychosom Med 2008 Sep;70(7):741-756.

19. Kavussanu M, McAuley E. Exercise and optimism: are highly active individuals more optimistic? Journal of Sport and Exercise Psychology 1995; 17:246-246.

20. Scheier ME, Carver CS. Dispositional optimism and physical well-being: The influence of generalized outcome expectancies on health. J Pers 1987;55(2):169-210. 
21. Rasmussen HN, PhD, Scheier MF, PhD, Greenhouse JB, PhD. Optimism and Physical Health: A Meta-Analytic Review. Annals of Behavioral Medicine 2009 Jun 2009;37(3):239-56.

22. Scheier, M. F., Carver, C. S., \& Bridges, M. W. (1994). Distinguishing optimism from neuroticism (and trait anxiety, self-mastery, and self-esteem): A reevaluation of the life orientation test. Journal of Personality and Social Psychology, 67(6), 1063.

23. Otero-López, J. M., Luengo, A., Romero, E., Gómez, J. A., \& Castro, C. (1998). Psicología de la personalidad. Manual de prácticas. Barcelona: Ariel Practicum.

24. Pender, N. (1996). Modelo de promoción de la salud. Health Promotion in Nursing. Practice.3. Ed. Connecticut: Appleton y Lange, 5175.

25. Walker, S. N., Kerr, M. J., Pender, N. J., \& Sechrist, K. R. (1990). A Spanish Language Version Of the Health-Promoting Lifestyle Profile. Nursing Research, 39(5), 268-273.

26. Brown JD. Likert items and scales of measurement. Shiken: JALT Testing \& Evaluation SIG Newsletter 2011;15(1):10-14.

27. Baggaley AR, Hull AL. The effect of nonlinear transformations on a Likert scale. Eval Health Prof 1983 Dec;6(4):483-491.

28. Altman DG, Bland JM. Statistics notes: the normal distribution. BMJ 1995 Feb 4;310(6975):298.

29. Ghasemi A, Zahediasl S. Normality tests for statistical analysis: a guide for non-statisticians. International journal of endocrinology and metabolism 2012;10(2):486-489.

30. Segerstrom SC. Optimism and resources: Effects on each other and on health over 10 years. Journal of Research in Personality 2007 8;41(4):772-786

31. Kelloniemi H, Ek E, Laitinen J. Optimism, dietary habits, body mass index and smoking among young Finnish adults. Appetite 2005 10;45(2):169-176.

32. Adams TB, Bezner JR. Conceptualization and Measurement of the Spiritual and Psychological Dimensions of Wellness in a. Journal of American College Health 2000 01;48(4):165.

33. Kaplan GA, Camacho T. Perceived health and mortality: a nine-year follow-up of the human population laboratory cohort. Am J Epidemiol 1983 Mar;117(3):292-304.

34. Baldwin DR, Chambliss LN, Towler K. Optimism and Stress: an African-American College Student Perspective. College Student Journal 2003 06;37(2):276.

35. Kiecolt-Glaser JK, Page GG, Marucha PT, MacCallum RC, Glaser R. Psychological influences on surgical recovery: perspectives from psychoneuroimmunology. Am Psychol 1998;53(11):1209.

36. Stellar JE, John-Henderson N, Anderson CL, Gordon AM, McNeil GD, Keltner D. Positive affect and markers of inflammation: Discrete positive emotions predict lower levels of inflammatory cytokines. Emotion 2015;15(2):129.

37. Jaimes EL, Díaz MPG. Estilos de vida saludable en estudiantes de Enfermería en la Universidad Cooperativa de Colombia. Revista Hacia la Promoción de la Salud 2014;19(1):68-83.

38. Wang $D$, Xing XH, Wu XB. Healthy lifestyles of university students in China and influential factors. ScientificWorldJournal 2013 Jul 9; 2013:412950.

39. Triviño-Vargas ZG. Conductas promotoras de salud en estudiantes de una institución de educación superior. Aquichan 2012;12(3):275-285.

40. Vidal Gutiérrez D, Chamblas García I, Zavala Gutiérrez M, MÜLLER GILCHRIST R, Rodríguez Torres MC, Chávez Montecino A. Determinantes Sociales en salud y estilos de vida en población adulta de concepción, chile. Ciencia y enfermería 2014;20(1):61-74.

41. Cid P, Merino JM, Stiepovich J. Factores biológicos y psicosociales predictores del estilo de vida promotor de salud. Revista médica de Chile 2006;134(12):1491-1499.

42. Shaheen A, Nassar O, Amre, H. and Hamdan-Mansour, A. Factors Affecting Health-Promoting Behaviors of University Students in Jordan. Health 2015;7(1):8-1-8. 


\section{Anexo:}

\section{Tablas y gráficos}

\begin{tabular}{|c|c|c|c|c|}
\hline & Frecuencia absoluta & Grupos & $\begin{array}{l}\text { Porcentaje } \\
\text { subgrupos }\end{array}$ & $\begin{array}{l}\text { Casos } \\
\text { perdidos }\end{array}$ \\
\hline \multirow[t]{4}{*}{ Edad (años) } & \multirow[t]{4}{*}{905} & $\leq 19$ & 40.4 & \multirow[t]{4}{*}{0} \\
\hline & & $20-25$ & 53.8 & \\
\hline & & $26-30$ & 2 & \\
\hline & & $\geq 31$ & 3.8 & \\
\hline \multirow[t]{2}{*}{ Género } & \multirow[t]{2}{*}{901} & Masculino & 26.4 & \multirow[t]{2}{*}{4} \\
\hline & & Femenino & 73.1 & \\
\hline \multirow[t]{4}{*}{ Estudios } & \multirow[t]{4}{*}{904} & Formación Profesional & 10.5 & \multirow[t]{4}{*}{1} \\
\hline & & Bachiller & 73.9 & \\
\hline & & Prueba acceso $>25$ años & 2.8 & \\
\hline & & Otra diplomatura o licenciatura & 12.7 & \\
\hline \multirow[t]{2}{*}{ Ocupación } & \multirow[t]{2}{*}{898} & Trabaja & 13.1 & \multirow[t]{2}{*}{7} \\
\hline & & No trabaja & 86.1 & \\
\hline \multirow[t]{3}{*}{ Estado civil } & \multirow[t]{3}{*}{905} & Soltero & 93 & \multirow[t]{3}{*}{0} \\
\hline & & Casado & 1.8 & \\
\hline & & En pareja & 5.2 & \\
\hline \multirow[t]{4}{*}{ Titulación } & \multirow[t]{4}{*}{905} & Enfermería Alcalá & 32 & \multirow[t]{4}{*}{0} \\
\hline & & Enfermería Guadalajara & 23 & \\
\hline & & Fisioterapia & 15.8 & \\
\hline & & Medicina & 29.2 & \\
\hline \multirow[t]{3}{*}{ Curso } & \multirow[t]{3}{*}{905} & $1 .^{\circ}$ & 36.2 & \multirow[t]{3}{*}{0} \\
\hline & & $2 .^{\circ}$ & 31.8 & \\
\hline & & $3 .^{\circ}$ & 31.9 & \\
\hline
\end{tabular}

Tabla 1: Estadísticos descriptivos de las variables sociodemográficas 


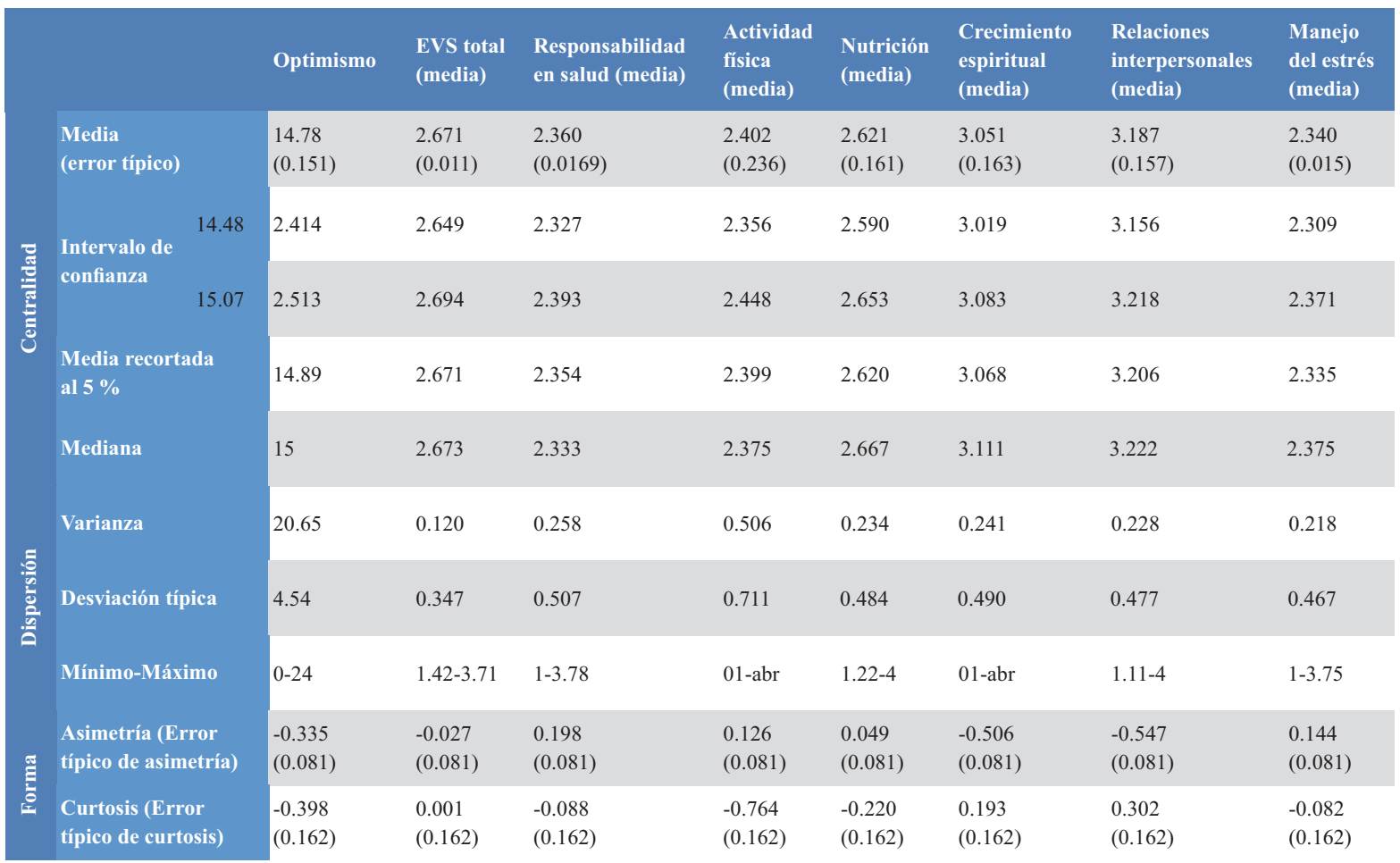

Tabla 2: Estadísticos descriptivos de los valores medios de las variables resultado 


\begin{tabular}{lccc} 
& \multicolumn{4}{c}{ Optimismo } \\
\cline { 2 - 4 } & $\mathrm{R}$ & $\mathrm{p}$ & $\mathrm{R} 2$ \\
\hline EVS global & 0.370 & 0.000 & 0.137 \\
\hline Responsabilidad en salud & 0.108 & 0.001 & 0.012 \\
\hline Actividad física & 0.147 & 0.000 & 0.022 \\
\hline Nutrición & 0.194 & 0.000 & 0.038 \\
\hline Crecimiento espiritual & 0.480 & 0.000 & 0.230 \\
\hline Relaciones interpersonales & 0.310 & 0.000 & 0.096 \\
\hline Manejo del estrés & 0.279 & 0.000 & 0.078
\end{tabular}

Siendo $R$ = coeficiente de correlación de Pearson;

$p=$ nivel de significancia; $R=$ coeficiente de determinación, calculado para $d f 1=1$ y d $2=903$.

Tabla 3: Correlación entre el nivel de optimismo y el EVS global y sus dimensiones

\begin{tabular}{|c|c|c|c|c|c|c|c|c|}
\hline & \multicolumn{3}{|c|}{ HOMBRES } & \multicolumn{3}{|c|}{ MUJERES } & \multirow{2}{*}{$\mathbf{t}$} & \multirow{2}{*}{$\mathbf{p}$} \\
\hline & MEDIA & DE & IC 95\% & MEDIA & DE & IC $95 \%$ & & \\
\hline Optimismo & 15.88 & 0.288 & $(15.32,16.45)$ & 14.38 & 0.176 & $(14.04,14.73)$ & 4.418 & 0.00 \\
\hline EVS global & 142.14 & 1.152 & $\begin{array}{l}\text { (139.87, } \\
144.41)\end{array}$ & 137.64 & 0.698 & $(136.27,139.01)$ & 3.327 & 0.001 \\
\hline Nutrición & 24.11 & 0.296 & $(23.53,24.70)$ & 23.40 & 0.166 & $(23.07,23.73)$ & 2.168 & 0.0 \\
\hline Actividad física & 21.07 & 0.380 & $(20.32,21.82)$ & 18.55 & 0.212 & $(18.14,18.97)$ & 5.983 & 0.0 \\
\hline $\begin{array}{l}\text { Relaciones } \\
\text { interpersonales }\end{array}$ & 28.18 & 0.269 & $(27.65,28.71)$ & 28.83 & 0.168 & $(28.50,29.16)$ & $\overline{-}-\overline{0}$ & 0.044 \\
\hline Manejo del estrés & 19.47 & 0.235 & $(19.01,19.94)$ & 18.43 & 0.145 & $(18.14,18.71)$ & 3.738 & 0.0 \\
\hline
\end{tabular}

Siendo F = estadistico F de Snedecor ; $p=$ nivel de significancia; $t=$ estadistico $t$ de Student .

Tabla 4: Análisis por género

\begin{tabular}{|c|c|c|c|c|c|c|c|c|c|c|c|c|c|c|c|}
\hline & \multicolumn{3}{|c|}{$\begin{array}{l}\text { ENFERMERÍA } \\
\text { ALCALÁ }\end{array}$} & \multicolumn{3}{|c|}{$\begin{array}{l}\text { ENFERMERÍA } \\
\text { GUADALAJARA }\end{array}$} & \multicolumn{3}{|c|}{ FISIOTERAPIA } & \multicolumn{3}{|c|}{ MEDICINA } & \multirow[t]{2}{*}{ F } & \multirow[t]{2}{*}{ W } & \multirow[t]{2}{*}{$\mathbf{p}$} \\
\hline & MEDIA & DE & IC $95 \%$ & MEDIA & DE & IC 95\% & MEDIA & DE & IC $95 \%$ & MEDIA & DE & IC $95 \%$ & & & \\
\hline Optimismo & 13.87 & 0.270 & $\begin{array}{l}(13.34 \\
14.40)\end{array}$ & 14.84 & 0.295 & $\begin{array}{l}(14.26 \\
15.42)\end{array}$ & 15.06 & 0.350 & $\begin{array}{l}(14.36 \\
15.75)\end{array}$ & 15.57 & 0.292 & $\begin{array}{l}(15.00 \\
16.15)\end{array}$ & 6.878 & & 0.000 \\
\hline EVS global & 137.46 & 1.146 & $\begin{array}{l}(135.20, \\
139.71)\end{array}$ & 140.53 & 1.178 & $\begin{array}{l}(138.21, \\
142.85)\end{array}$ & 138.43 & 1.348 & $\begin{array}{l}(135.77 \\
141.10)\end{array}$ & 139.25 & 1.115 & $\begin{array}{l}(137.05, \\
141.44)\end{array}$ & & 1.235 & 0.297 \\
\hline Nutrición & 23.61 & 0.268 & $\begin{array}{l}(23.09 \\
24.14)\end{array}$ & 23.90 & 0.278 & $\begin{array}{l}(23.35 \\
24.45)\end{array}$ & 23.48 & 0.360 & $\begin{array}{l}(22.77 \\
24.19)\end{array}$ & 23.37 & 0.272 & $\begin{array}{l}(22.83 \\
23.90)\end{array}$ & 0.609 & & 0.609 \\
\hline Actividad física & 18.42 & 0.340 & $\begin{array}{l}(17.75, \\
19.09)\end{array}$ & 19.47 & 0.365 & $\begin{array}{l}(18.75 \\
20.19)\end{array}$ & 20.17 & 0.482 & $\begin{array}{l}(19.22, \\
21.13)\end{array}$ & 19.35 & 0.357 & $\begin{array}{l}(18.65 \\
20.06)\end{array}$ & 3.468 & & 0.016 \\
\hline \begin{tabular}{|l} 
Relaciones \\
interpersonales
\end{tabular} & 28.71 & 0.259 & $\begin{array}{l}(28.80 \\
29.22)\end{array}$ & 29.00 & 0.301 & $\begin{array}{l}(28.41, \\
29.59)\end{array}$ & 28.01 & 0.311 & $\begin{array}{l}(27.39 \\
28.62)\end{array}$ & 28.71 & 0.272 & $\begin{array}{l}(28.17 \\
29.24)\end{array}$ & 1.559 & & 0.198 \\
\hline $\begin{array}{l}\text { Manejo } \\
\text { del estrés }\end{array}$ & 18.45 & 0.233 & $\begin{array}{l}(17.99, \\
18.91)\end{array}$ & 18.71 & 0.239 & $\begin{array}{l}(18.24, \\
19.18)\end{array}$ & 18.85 & 0.279 & $\begin{array}{l}(18.30 \\
19.40)\end{array}$ & 18.92 & 0.240 & $\begin{array}{l}(18.44, \\
19.39)\end{array}$ & & 0.739 & 0.529 \\
\hline
\end{tabular}

Siendo $F=$ estadistico $F$ de Snedecor $;$ w=estadistico Welch; $p=$ nivel de significancia .

Tabla 5: Análisis por titulación 


\begin{tabular}{|c|c|c|c|c|c|c|}
\hline Optimismo & & & $\mathbf{p}$ & & & p \\
\hline \multirow[t]{6}{*}{ Turkey } & $\begin{array}{l}\text { Enfermería } \\
\text { Alcalá }\end{array}$ & $\begin{array}{l}\text { Enfermería } \\
\text { Guadalajara }\end{array}$ & 0.082 Cabriel & $\begin{array}{l}\text { Enfermería } \\
\text { Alcalá }\end{array}$ & $\begin{array}{l}\text { Enfermería } \\
\text { Guadalajara }\end{array}$ & 0.099 \\
\hline & & Fisioterapia & 0.049 & & Fisioterapia & 0.052 \\
\hline & & Medicina & 0.000 & & Medicina & 0.000 \\
\hline & $\begin{array}{l}\text { Enfermería } \\
\text { Guadalajara }\end{array}$ & Fisioterapia & 0.972 & $\begin{array}{l}\text { Enfermería } \\
\text { Guadalajara }\end{array}$ & Fisioterapia & 0.998 \\
\hline & & Medicina & 0.298 & & Medicina & 0.392 \\
\hline & Fisioterapia & Medicina & 0.687 & Fisioterapia & Medicina & 0.841 \\
\hline Actividad física & & & $\mathrm{p}$ & & & p \\
\hline \multirow[t]{6}{*}{ Turkey } & $\begin{array}{l}\text { Enfermería } \\
\text { Alcalá }\end{array}$ & $\begin{array}{l}\text { Enfermería } \\
\text { Guadalajara }\end{array}$ & 0.173 & $\begin{array}{l}\text { Enfermería } \\
\text { Alcalá }\end{array}$ & $\begin{array}{l}\text { Enfermería } \\
\text { Guadalajara }\end{array}$ & 0.219 \\
\hline & & Fisioterapia & 0.013 & & Fisioterapia & 0.013 \\
\hline & & Medicina & 0.213 & & Medicina & 0.278 \\
\hline & $\begin{array}{l}\text { Enfermería } \\
\text { Guadalajara }\end{array}$ & Fisioterapia & 0.664 & $\begin{array}{l}\text { Enfermería } \\
\text { Guadalajara }\end{array}$ & Fisioterapia & 0.824 \\
\hline & & Medicina & 0.996 & & Medicina & 1.000 \\
\hline & Fisioterapia & Medicina & 0.502 & Fisioterapia & Medicina & 0.643 \\
\hline
\end{tabular}

Siendo $p=$ nivel de significancia .

Tabla 6: Análisis comparaciones múltiples por titulación

\begin{tabular}{|c|c|c|c|c|c|c|c|c|c|c|c|c|}
\hline & \multicolumn{3}{|c|}{$1^{0}$} & \multicolumn{3}{|c|}{$2^{0}$} & \multicolumn{3}{|c|}{$3^{\circ}$} & \multirow{2}{*}{$\mathbf{F}$} & \multirow{2}{*}{ W } & \multirow{2}{*}{$\mathbf{p}$} \\
\hline & MEDIA & DE & IC $95 \%$ & MEDIA & DE & IC $95 \%$ & MEDIA & DE & IC $95 \%$ & & & \\
\hline Optimismo & 14.63 & 0.256 & $\begin{array}{l}(14.12 \\
15.13)\end{array}$ & 14.88 & 0.270 & $\begin{array}{l}(14.34 \\
15.41)\end{array}$ & 14.85 & 4.418 & $\begin{array}{l}(14.34 \\
15.36)\end{array}$ & 0.289 & & 0.749 \\
\hline EVS global & 136.94 & 0.944 & $\begin{array}{l}(135.08, \\
138.80)\end{array}$ & 140.52 & 1.027 & $\begin{array}{l}(138.50, \\
142.55)\end{array}$ & 139.31 & 1.146 & $\begin{array}{l}(137.06, \\
141.57)\end{array}$ & & 3.440 & 0.033 \\
\hline Nutrición & 23.29 & 0.243 & $\begin{array}{l}(22.81 \\
23.76)\end{array}$ & 23.58 & 0.255 & $\begin{array}{l}(23.08 \\
24.08)\end{array}$ & 23.93 & 0.254 & $\begin{array}{l}(23.43, \\
24.43)\end{array}$ & 1.700 & & 0.183 \\
\hline $\begin{array}{l}\text { Relaciones } \\
\text { interpersonales }\end{array}$ & 28.48 & 0.240 & $\begin{array}{l}(28.01 \\
28.95)\end{array}$ & 29.07 & 0.244 & $\begin{array}{l}(28.59, \\
29.55)\end{array}$ & 28.47 & 0.258 & $\begin{array}{l}(27.97, \\
28.98)\end{array}$ & 1.869 & & 0.155 \\
\hline $\begin{array}{l}\text { Manejo } \\
\text { del estrés }\end{array}$ & 18.21 & 0.191 & $\begin{array}{l}(17.84, \\
18.59)\end{array}$ & 19.14 & 0.209 & $\begin{array}{l}(18.72, \\
19.55)\end{array}$ & 18.85 & 0.243 & $\begin{array}{l}(18.37, \\
19.33)\end{array}$ & & 5.573 & 0.004 \\
\hline
\end{tabular}

Siendo $F=$ estadístico $F$ de Snedecor ; $w=$ estadístico Welch; $p=$ nivel de significancia.

Tabla 7: Análisis por edad

\begin{tabular}{|c|c|c|c|c|c|c|c|}
\hline Optimismo & & & $\mathbf{p}$ & Actividad física & & & $\mathbf{P}$ \\
\hline \multirow[t]{6}{*}{ Gabriel } & $\leq 19$ AÑOS & 20-25 AÑOS & 0.316 & Gabriel & $\leq 19$ AÑOS & 20-25 AÑOS & 0.694 \\
\hline & & 26-30 AÑOS & 0.999 & & & 26-30 AÑOS & 0.342 \\
\hline & & $\geq 31$ AÑOS & 0.012 & & & $\geq 31$ AÑOS & 0.001 \\
\hline & 20-25 AÑOS & 26-30 AÑOS & 1.000 & & 20-25 AÑOS & 26-30 AÑOS & 0.645 \\
\hline & & $\geq 31$ AÑOS & 0.109 & & & $\geq 31$ AÑOS & 0.004 \\
\hline & 26-30 AÑOS & $\geq 31$ AÑOS & 0.629 & & 26-30 AÑOS & $\geq 31$ AÑOS & 0.947 \\
\hline \multicolumn{2}{|c|}{ Relaciones interpersonales } & & p & & & & \\
\hline \multirow[t]{2}{*}{ Gabriel } & $\leq 19$ AÑOS & 20-25 AÑOS & 0.430 & & & & \\
\hline & & 26-30 AÑOS & 0.038 & & & & \\
\hline
\end{tabular}

Tabla 8: Análisis comparaciones múltiples por edad 


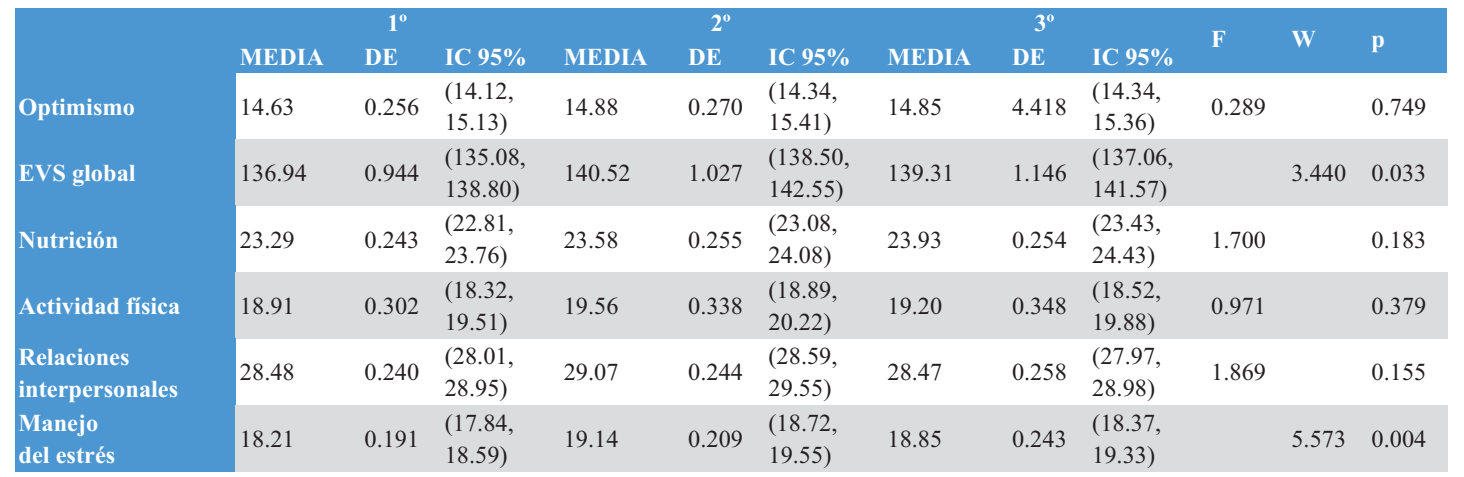

Siendo $F=$ estadistico $F$ de Snedecor ; $w=$ estadistico Welch; $p=$ nivel de significancia.

Tabla 9: Análisis por curso

\begin{tabular}{lccccccc} 
EVS global & \multicolumn{2}{c}{$\mathbf{p}$} & Manejo del estrés & \multicolumn{2}{c}{$\mathbf{P}$} \\
Games-Howell & $1^{\circ}$ & $2^{\circ}$ & 0.028 & Games-Hlowell & $1^{\circ}$ & $2^{\circ}$ & 0.003 \\
& & $3^{\circ}$ & 0.248 & & $3^{\circ}$ & 0.099 \\
& $2^{\circ}$ & $3^{\circ}$ & 0.711 & $2^{\circ}$ & $3^{\circ}$ & 0.650
\end{tabular}

Siendo $p=$ nivel de significancia.

Tabla 10: Análisis comparaciones múltiples por curso

\begin{tabular}{|c|c|c|c|c|c|c|}
\hline & $\begin{array}{l}\text { Responsabilidad } \\
\text { salud }\end{array}$ & $\begin{array}{l}\text { Actividad } \\
\text { física }\end{array}$ & Nutrición & $\begin{array}{l}\text { Crecimiento } \\
\text { espiritual }\end{array}$ & $\begin{array}{l}\text { Relaciones } \\
\text { interpersonales }\end{array}$ & $\begin{array}{l}\text { Manejo } \\
\text { del estrés }\end{array}$ \\
\hline $\begin{array}{l}\text { Responsabilidad } \\
\text { salud }\end{array}$ & 1 & & & & & \\
\hline Actividad física & 0.330 & 1 & & & & \\
\hline Nutrición & 0.361 & 0.402 & 1 & & & \\
\hline $\begin{array}{l}\text { Crecimiento } \\
\text { espiritual }\end{array}$ & 0.307 & 0.233 & 0.241 & 1 & & \\
\hline $\begin{array}{l}\text { Relaciones } \\
\text { interpersonales }\end{array}$ & 0.360 & 0.147 & 0.254 & 0.557 & 1 & \\
\hline $\begin{array}{l}\text { Manejo } \\
\text { del estrés }\end{array}$ & 0.362 & 0.391 & 0.269 & 0.482 & 0.325 & 1 \\
\hline
\end{tabular}

La correlación es significativa en todos los casos al nivel 0.01 (bilateral).

Tabla 11: Correlación (según coeficiente R de Pearson) de las dimensiones de EVS 


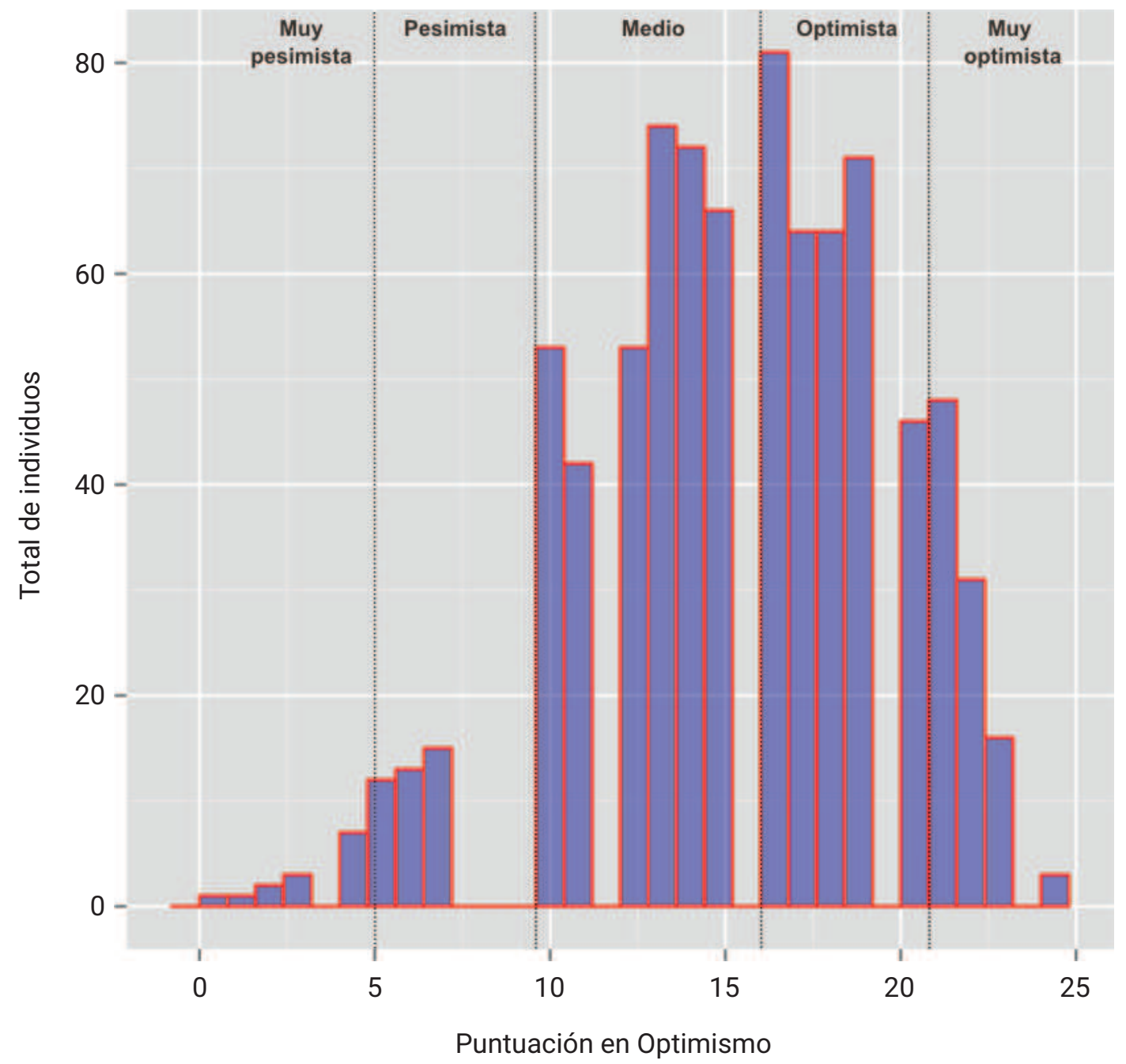

Gráfico 1: histograma de las respuestas al cuestionario LOT-R 


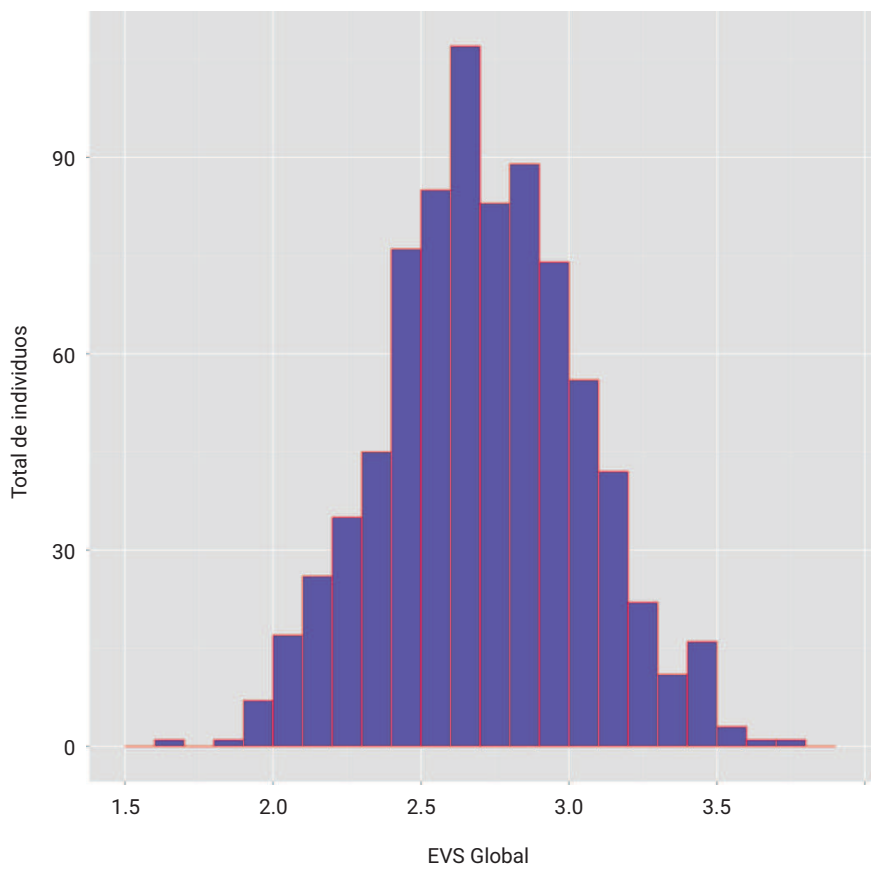

$1=$ Nunca; $2=$ A veces; $3=$ Con frecuencia; $4=$ Siempre

Gráfico 2: histograma de las respuestas al cuestionario HPLP II (global)

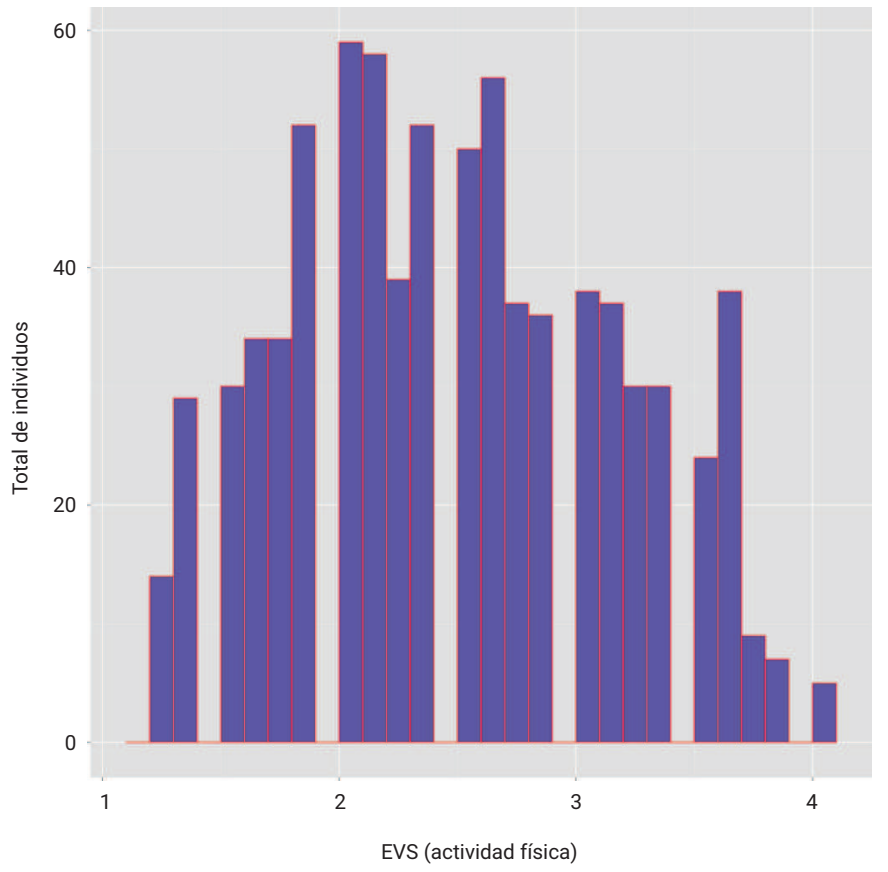

$1=$ Nunca; $2=A$ veces; $3=$ Con frecuencia; $4=$ Siempre

Gráfico 3: histograma de las respuestas al cuestionario HPLP II (subescala "actividad física") 


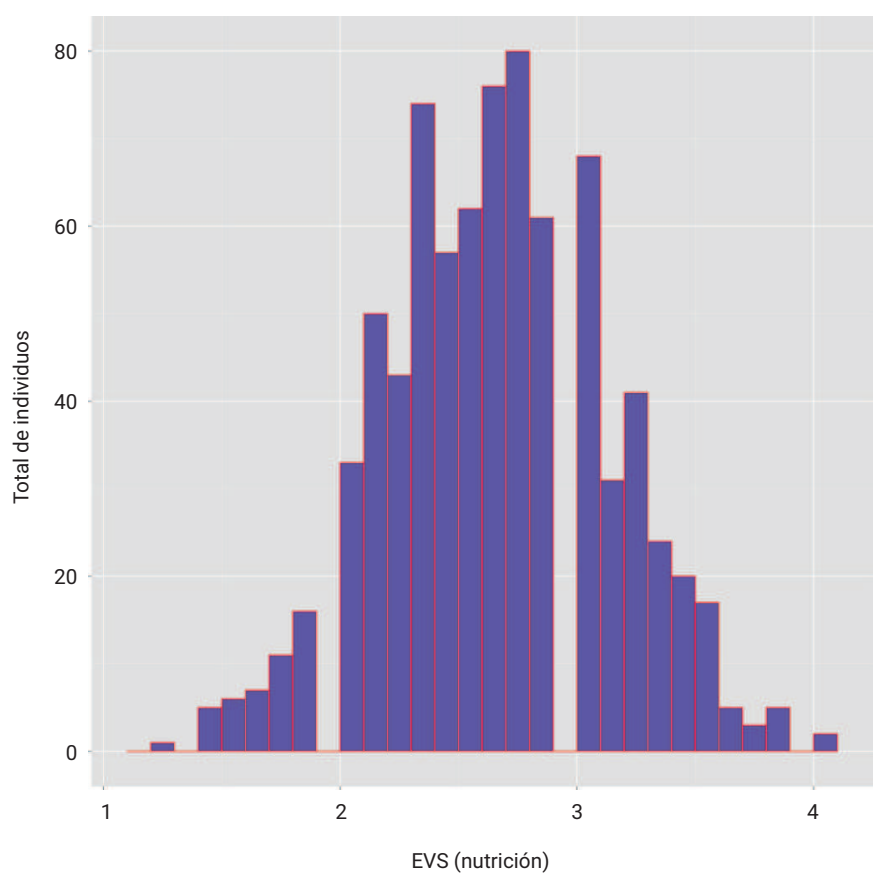

$1=$ Nunca; $2=$ A veces; $3=$ Con frecuencia; $4=$ Siempre

Gráfico 4: histograma de las respuestas al cuestionario HPLP II (subescala "nutrición")

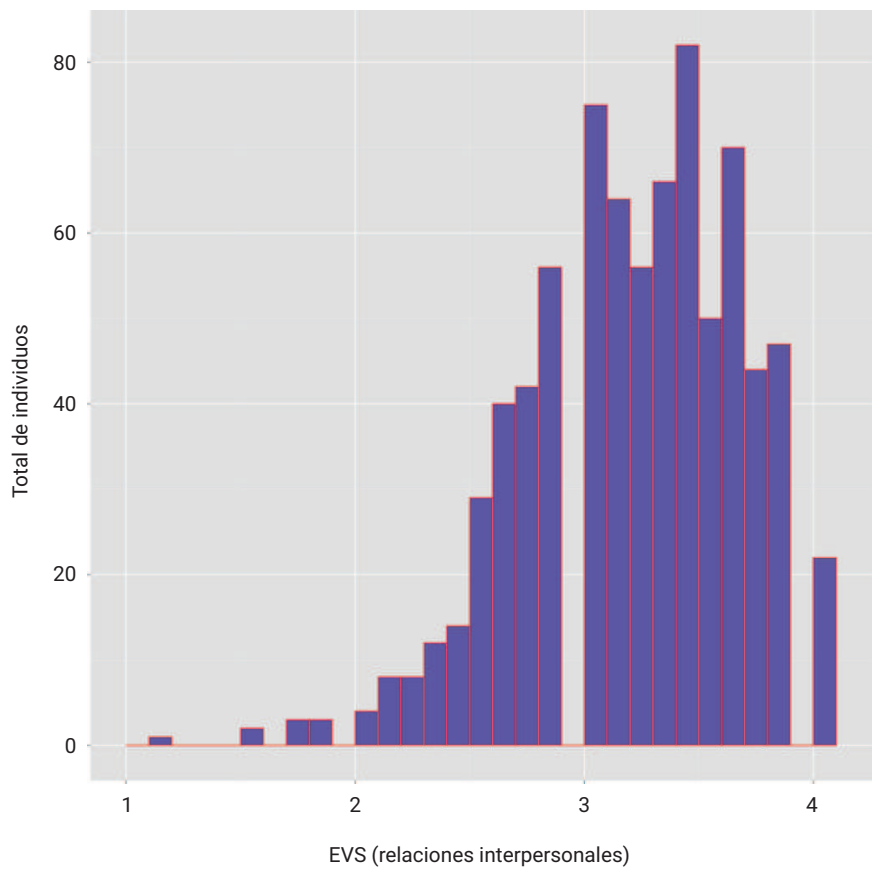

$1=$ Nunca; $2=A$ veces $3=$ Con frecuencia $;=$ Siempre

Gráfico 5: histograma de las respuestas al cuestionario HPLP II (subescala "relaciones interpersonales") 


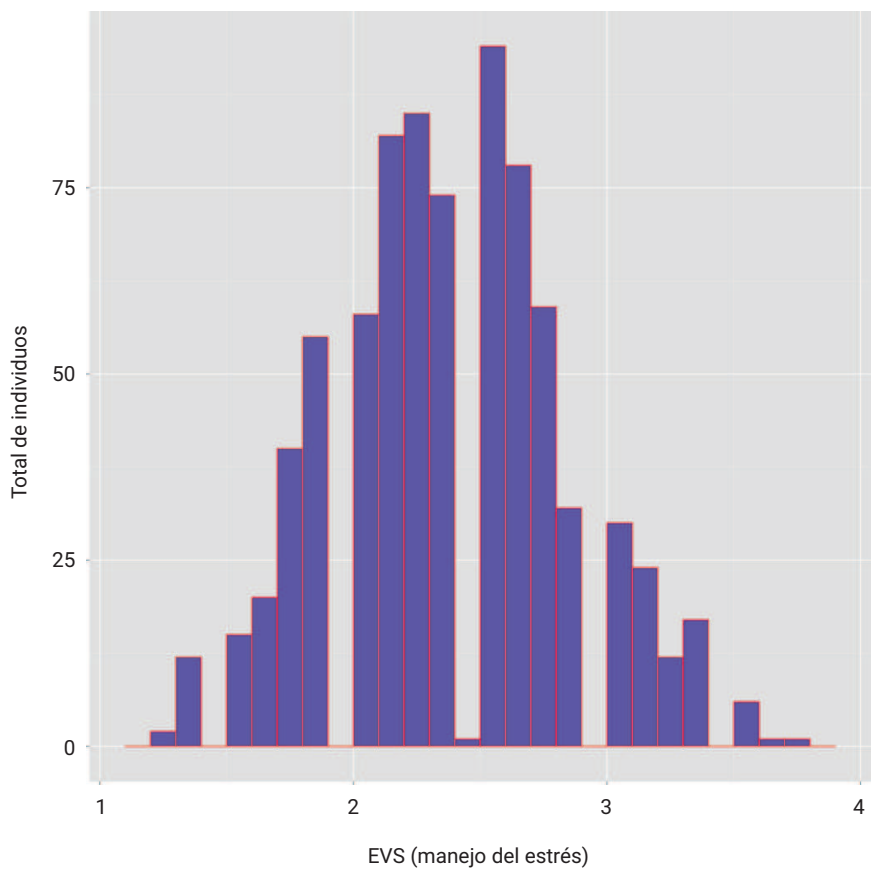

$1=$ Nunca; $2=\mathrm{A}$ veces; $3=$ Con frecuencia; $4=$ Siempre

Gráfico 6: histograma de las respuestas al cuestionario HPLP II (subescala "manejo del estrés")

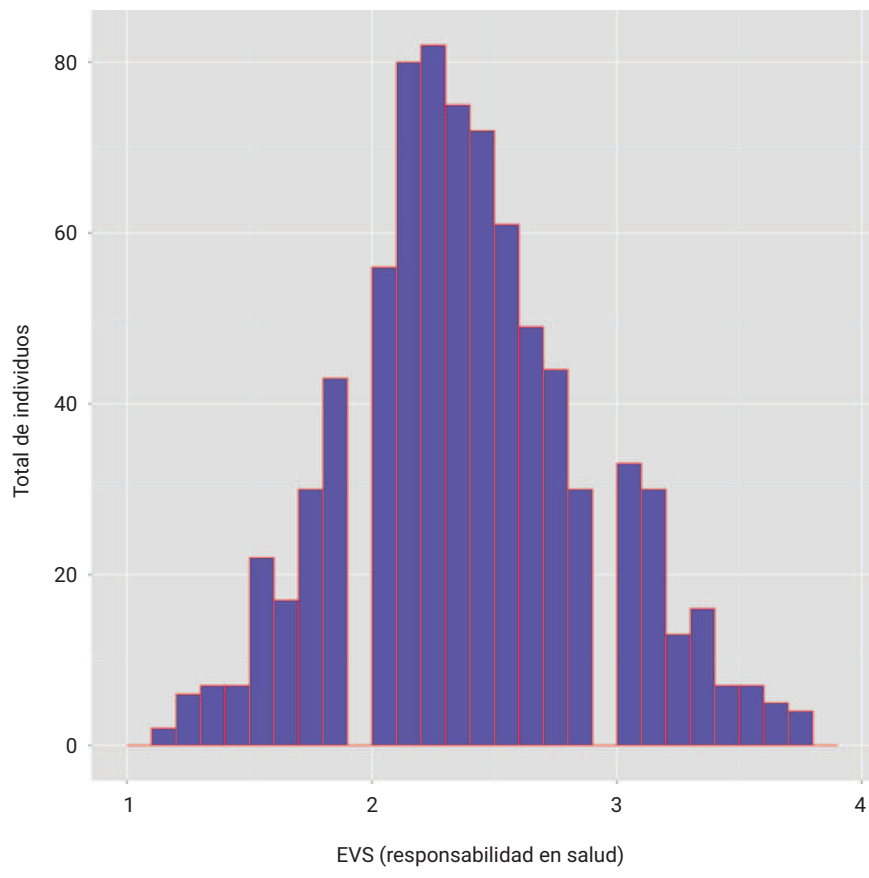

$1=$ Nunca; $2=\mathrm{A}$ veces; $3=$ Con frecuencia; $4=$ Siempre

Gráfico 7: histograma de las respuestas al cuestionario HPLP II (subescala "responsabilidad en salud") 


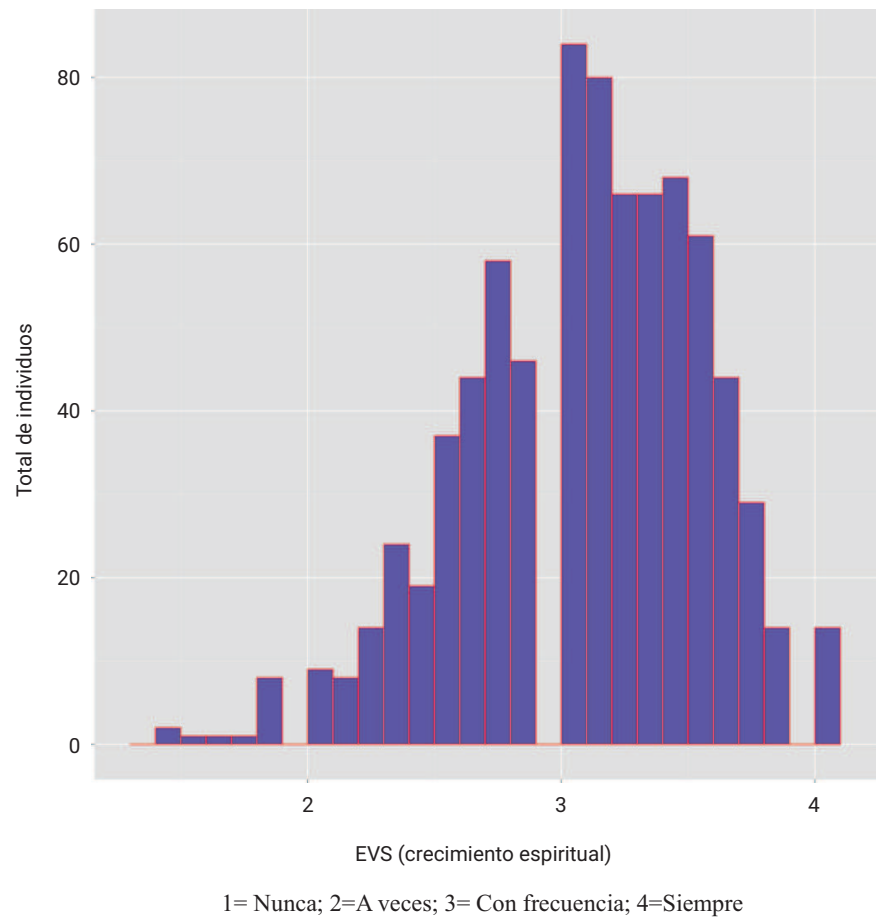

Gráfico 8: histograma de las respuestas al cuestionario HPLP II (subescala "crecimiento espiritual") 


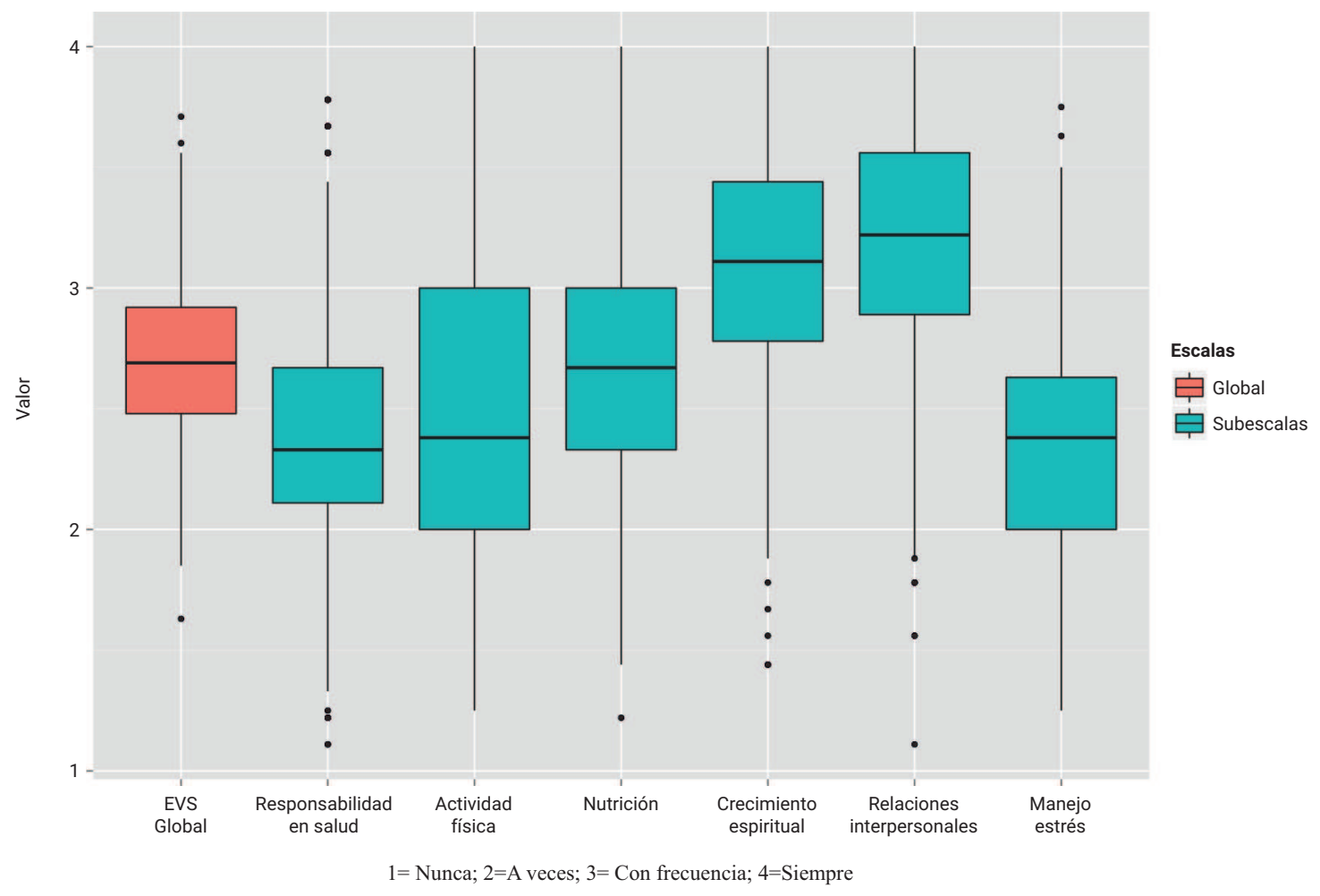

Gráfico 9: boxplot de distribución de los valores de EVS Global y sus subescalas 